\title{
Treatment of dengue fever
}

This article was published in the following Dove Press journal:

Infection and Drug Resistance

19 July 2012

Number of times this article has been viewed

\author{
Senaka Rajapakse ${ }^{1,2}$ \\ Chaturaka Rodrigo' \\ Anoja Rajapakse 3 \\ 'Department of Clinical Medicine, \\ Faculty of Medicine, University of \\ Colombo, Sri Lanka; ${ }^{2}$ Lincoln County \\ Hospital, United Lincolnshire NHS \\ Trust, Lincoln, UK; ${ }^{3}$ Kings Mill \\ Hospital, Sherwood Forest NHS \\ Foundation Trust, Mansfield, UK
}

Correspondence: Senaka Rajapakse Department of Clinical Medicine, Faculty of Medicine, University of Colombo 25, Kynsey Road, Colombo 08, Sri Lanka Tel +94I I2695300 ext I34 Fax +94I 12689188

Email senaka.ucfm@gmail.com
Abstract: The endemic area for dengue fever extends over 60 countries, and approximately 2.5 billion people are at risk of infection. The incidence of dengue has multiplied many times over the last five decades at an alarming rate. In the endemic areas, waves of infection occur in epidemics, with thousands of individuals affected, creating a huge burden on the limited resources of a country's health care system. While the illness passes off as a simple febrile episode in many, a few have a severe illness marked by hypovolemic shock and bleeding. Iatrogenic fluid overload in the management may further complicate the picture. In this severe form dengue can be fatal. Tackling the burden of dengue is impeded by several issues, including a lack of understanding about the exact pathophysiology of the infection, inability to successfully control the vector population, lack of specific therapy against the virus, and the technical difficulties in developing a vaccine. This review provides an overview on the epidemiology, natural history, management strategies, and future directions for research on dengue, including the potential for development of a vaccine.

Keywords: dengue, treatment, fluid resuscitation

\section{Introduction}

Dengue is a febrile illness that can have a fatal outcome in severe cases. It is a caused by a flavivirus with four distinct serotypes (DV-1, DV-2, DV-3, and DV-4). ${ }^{1}$ The virus is spread between humans by mosquito vectors of the Aedes genus, ie, Aedes aegypti and Aedes albopictus. ${ }^{2}$ The exact pathophysiology of severe dengue infection (dengue hemorrhagic fever and dengue shock syndrome) is still an enigma, although it is now widely accepted that the host immune system, host genetic makeup, and pathogen virulence all contribute towards the rapid deterioration seen in some patients. ${ }^{3}$

The incidence of dengue is rising and the disease is a major public health problem in the tropics. Dengue epidemics closely follow seasonal climatic change with waves of epidemics following each rainy season. During an epidemic, thousands may be affected. ${ }^{4,5}$ Whilst most of them recover from a simple febrile illness, a small but significant proportion go on to develop the dengue shock state with associated fatalities. In many affected areas, this adds up to a significant case fatality rate, predominantly among young children and individuals of working age. The difficulty in controlling dengue infection stems from three root causes, ie, the presence of four different serotypes of virus, each with the independent ability to cause fatal disease, a lack of understanding of pathophysiology, nonavailability of specific treatment, nonavailability of a vaccine for prevention, and difficulties in controlling the vector population. 
There is no specific treatment for dengue other than supportive measures and judicious fluid therapy. Clinical trials have assessed various therapeutic options with minimal success over the last 50 years. ${ }^{6,7}$ The danger period of dengue or the so-called "critical period" where the patient might undergo sudden deterioration is relatively short and is between 48-72 hours. If the patient is appropriately managed during this phase, the chance of a fatal outcome is minimal. Still, this requires close monitoring and frequent review of fluid management. If the patient recovers, there are no sequelae in uncomplicated dengue (rare complications of dengue, such as orchitis, oophorotis, keratitis, and encephalitis, can have a long-term impact in a very small minority of cases). ${ }^{2}$ Infection does provide temporary protection against the same serotype but not against other dengue virus serotypes. ${ }^{8}$

The case fatalities in severe dengue are attributable to several factors. During epidemics, hospitals are overburdened with large numbers of patients, and close monitoring and management is extremely difficult given the limited resources in developing countries. In addition, the lack of understanding of the exact pathophysiology of dengue has led to a paucity of sufficient evidence-based management protocols aimed at specific pathophysiological phenomena of the illness. Many patients, due to lack of awareness, present late to hospital, sometimes in shock; their management is difficult, with a poor outcome.

Due to practical difficulties in managing an established dengue infection, much emphasis is now placed on the prevention of transmission, by controlling vector populations. Various methods of vector control, ie, physical (removal of breeding places), chemical (insecticides and larvicides), and biological (use of bacteria such as Bacillus thuringiensis) have been used to control the transmission of disease. ${ }^{2}$ However, this is a difficult task because the mosquito breeds even in small quantities of clear stagnant water (for example, between the trunk and the leaves of plants with large foliage). Such habitats are almost impossible to eradicate.

Despite these barriers, certain therapeutic and preventive measures have been explored in the last few decades to minimize the disease burden of dengue and to avoid fatalities. This review provides an update on dengue with regard to several important aspects of the disease, including its epidemiology, natural history, and management, as well as avenues for future research.

\section{Materials and methods}

A MEDLINE search was performed for all articles with the key word "dengue" in the title and "management", "treatment", "guidelines", "vaccine", "corticosteroids", and "immunoglobulin" in the abstract. The initial search yielded 1121 hits on these criteria. The search was then restricted to articles published in the English language within the last two decades (1992-2012). Endnote X3 software (Thomson Reuters, Carlsbad, CA) was used to filter articles. Bibliographies of cited literature were also searched. All abstracts were read independently by the three authors, and key articles were identified based on a consensus among the authors. Fifty-three sources were selected for final synthesis based on their relevance to the subtopics. The search was restricted to the last two decades to avoid redundant data and to select more recent evidence. However, related or cited papers of crucial trials before this period have also been included. The epidemiological data and guidelines for treatment were downloaded from the websites of international agencies, including the World Health Organization.

\section{Overview of epidemiology and natural history}

As mentioned above, dengue is an endemic vector-borne disease predominantly seen in the tropics. Cases have been reported across the continents of Asia, Africa, The Americas, Europe, and Australia. The World Health Organization estimates that 2.5 billion people worldwide live in dengueendemic areas, and 50 million new infections occur annually. ${ }^{9}$ There has been a significant rise in the number of epidemics and reported cases of dengue fever over the last 50 years. This represents an increase in detection rates with improved reporting, plus a true increase in incidence due to changes in environmental, climatic factors, and man-vector interaction. From 1955-1959 the average annual number of dengue infections reported to the World Health Organization was just 908 from less than 10 countries. From 2000-2007, the reported number was as high as 925,896 from more than 60 countries. ${ }^{9}$ Thus, the incidence of infection has been rising at an alarming rate. The distribution of disease burden within the endemic belt is also asymmetrical. Approximately $75 \%$ of the world's dengue burden is in South East Asian and Western Pacific regions. ${ }^{9}$ However, with increased numbers of travelers in and out of endemic areas, dengue infections are increasingly encountered by physicians in nonendemic areas as well. Hence the disease burden is no longer limited to "endemic" areas. ${ }^{10}$

The natural history of dengue infection is fairly straightforward. The incubation period following inoculation of the virus is around 4-7 days. ${ }^{11}$ The symptomatic phase of the illness is divided into three phases, ie, a febrile phase, a critical phase, and a recovery phase. ${ }^{9}$ 
The febrile phase lasts for 5-7 days and is characterized by high fever, retro-orbital headache, arthralgia, myalgia, malaise, nausea, and vomiting. ${ }^{9}{ }^{12}$ Examination may reveal an inflamed throat, lymphadenopathy, and tender hepatomegaly towards the latter part of the febrile phase. In many patients, the febrile phase is followed by the recovery phase and the entire illness may pass off as a simple febrile episode. The first diagnostic factor towards dengue at this stage may be the progressive drop in platelet count. It is commonly observed that the platelet count can drop rapidly from values above $250,000 / \mu \mathrm{L}$ to less than $100,000 / \mu \mathrm{L}$ over $2-3$ days. If a drop in platelet count is seen in serial full blood counts, it is recommended to observe any patient with fever over the next $3-5$ days with daily blood counts, especially in endemic areas during the times of epidemics. IgM antibodies against the virus are detectable in serum 5 days after the onset of fever. ${ }^{11}$

The critical phase can start at any time from 3-7 days since the onset of the fever. The pathophysiological hallmark of the critical phase is increased capillary permeability with extravasation of fluids. ${ }^{13}$ As mentioned above, the exact mechanism for this capillary leakage is unclear, though immune mechanisms are thought to play a major role. Although the maximum drop in platelet count is usually seen during the critical phase, it is only a surrogate marker for the severity of illness. In some patients, the drop in platelet count can be as low as below $5000 / \mu \mathrm{L}$. The onset of the critical phase is determined by evidence of fluid leakage only. ${ }^{14}$ This includes either clinically or radiologically demonstrable pleural effusion or ascites and/or evidence of hemoconcentration as shown by an increasing packed cell volume in serial blood counts. The critical phase lasts for 24-48 hours. During this time, the fluid balance must be carefully adjusted to strike a balance between keeping the vascular compartment adequately filled in the face of leakage while avoiding fluid overload later on. The fever characteristically comes down at the onset of the critical phase. Therefore defervescence is not a reason for complacency in dengue and only calls for more intense monitoring in the coming days.

Shock can occur during the critical phase for two reasons, ie, increased fluid leakage without an adequate intake and internal bleeding (facilitated by thrombocytopenia and deranged clotting). In the former situation, the packed cell volume will rise and in the latter it will fall. ${ }^{9}$ The typical signs of hypovolemic shock may be apparent in severe disease, but it is of vital importance to identify an at-risk patient well in advance. Serial monitoring for evidence of fluid leakage to approximate accurately the onset of the critical phase, close monitoring during the critical phase to identify bleeding (mucosal and skin bleeds, abdominal pain, melena, hematemesis, per rectal bleeding), monitoring of clinical parameters such as postural drop in blood pressure, postural tachycardia, and urine output to appreciate the fullness of the intravascular compartment, will all help to avoid death in severe dengue. ${ }^{9}$

If the patient survives the critical phase he or she will enter the recovery phase. At this time (48-72 hours following the critical phase), the extravasated fluid will re-enter the intravascular compartment ${ }^{9}$ and, if there had been overjudicious administration of fluids, the patient could develop heart failure or pulmonary edema. ${ }^{15}$ Death can occur due to fluid overload as much as it can due to shock from bleeding or leakage. If careful fluid management has taken place, recovery will be obvious at the bedside, because the patient feels better, regains their appetite, and becomes more active. An itchy erythematous rash with speckles of white in between is observed in some patients during convalescence. ${ }^{16}$ The platelet count rises rapidly at this time and can easily cross the $150,000 / \mu \mathrm{L}$ mark in $2-3$ days from values well below $50,000 / \mu \mathrm{L}$.

What is described above is the typical natural history of a dengue infection. There can be many variations in the progression of illness with each patient. Some may have prolonged periods of thrombocytopenia while others may have a prolonged febrile phase. In some instances, fever may continue into the critical phase. Allowances must be made for individual variations due to host (immunity, comorbidities) and virus (virulence) related factors.

\section{Management of dengue: an overview Symptomatic management}

During the febrile phase, liberal oral fluid administration and antipyretic treatment with paracetamol as required is recommended. ${ }^{9}$ Other nonsteroidal anti-inflammatory drugs should be avoided. If the patient has access to a health care facility nearby, he or she can be managed at home with observation of daily full blood counts. Excessive vomiting or diarrhea resulting in dehydration, severe prostration, or early bleeding manifestations are all indications for admission to hospital for close observation.

\section{Management of the critical phase}

No specific therapy has been shown to be effective in the treatment of any of the manifestations of dengue in a randomized controlled trial (Table 1). The mainstay of treatment is still careful fluid management. 
Table I Summary of evidence of benefit for available intervention in dengue infection

\begin{tabular}{|c|c|}
\hline Intervention & Conclusion \\
\hline $\begin{array}{l}\text { Choice of } \\
\text { intravenous fluid }\end{array}$ & $\begin{array}{l}\text { RCTs show no benefit of colloids over } \\
\text { crystalloids, }{ }^{17-19} \text { although a trend towards benefit is } \\
\text { seen with colloids over crystalloids in severe cases }\end{array}$ \\
\hline $\begin{array}{l}\text { Rate of fluid } \\
\text { infusion }\end{array}$ & $\begin{array}{l}\text { No evidence available, regimens based on experience } \\
\text { of centers treating large numbers of cases }\end{array}$ \\
\hline $\begin{array}{l}\text { Transfusion of } \\
\text { blood products }\end{array}$ & $\begin{array}{l}\text { Small RCT suggests that fresh frozen plasma may } \\
\text { increase platelet counts }{ }^{21}\end{array}$ \\
\hline Nasal CPAP & $\begin{array}{l}\text { RCT shows benefit in terms of improvement } \\
\text { of hypoxemia and reduction of the need for } \\
\text { ventilation in patients with dengue and acute } \\
\text { respiratory failure }{ }^{33}\end{array}$ \\
\hline $\begin{array}{l}\text { Carbazochrome } \\
\text { sodium sulfonate }\end{array}$ & $\begin{array}{l}\text { RCT shows no evidence of benefit, but } \\
\text { underpowered } \\
\text { 35 }\end{array}$ \\
\hline Corticosteroids & $\begin{array}{l}\text { Systematic review of RCTs shows no benefit, but } \\
\text { available RCT evidence is from studies } \\
\text { performed }>20 \text { years ago; }{ }^{25,26} \text { case series provide } \\
\text { limited evidence of possible benefit in severe dengue }\end{array}$ \\
\hline Immunoglobulins & $\begin{array}{l}\text { Single RCT shows no benefit for } \\
\text { thrombocytopenia; }{ }^{54} \text { no evidence available } \\
\text { for other manifestations }{ }^{55}\end{array}$ \\
\hline
\end{tabular}

Abbreviations: RCT, randomized controlled trial; CPAP, continuous positive airways pressure.

\section{Fluid resuscitation}

The key issue here is the prediction and identification of the onset of the critical phase. When an effusion or ascites becomes clinically detectable, it indicates that the critical phase has already started several hours ago. ${ }^{14} \mathrm{~A}$ lateral decubitus chest radiograph or an ultrasound scan can be useful to detect early signs of fluid accumulation in serous cavities, but may not be technically feasible for each and every patient at times of epidemics. ${ }^{14}$ Generally, the onset of the critical phase can be predicted when the hematocrit starts rising above baseline (note that many patients are admitted to hospital well into the critical phase and there is a dilemma as to their "normal" hematocrit). A drop in platelet count to less than $100,000 / \mu \mathrm{L}$ is also an indicator that the patient is at risk of entering the critical phase in the next 24 hours. ${ }^{14}$

The next step in management is close observation of vital parameters, fullness of the vascular system (postural drop in blood pressure, postural tachycardia), clinical evidence of hypovolemic shock, and manifestations of bleeding. Monitoring charts (those that graphically indicate key parameters such as hematocrit and platelet count) must be accurately maintained. Regular reviews (at least four-hourly) by physicians and nurses are essential during the critical phase.

The key management strategy during the critical phase is judicious fluid administration. ${ }^{9}$ The evidence base regarding the best choice of fluid, or the amount of fluid which should be administered, is limited. For obvious ethical reasons, there are no trials comparing the administration of intravenous fluids against placebo. Fluids used for volume expansion include normal saline, Ringer lactate, $5 \%$ glucose diluted $1: 2$ or 1:1 in normal saline, plasma, plasma substitutes, or $5 \%$ albumin. Currently there is no evidence that colloids are either superior or inferior to crystalloids. A series of studies in Vietnam have compared the use of crystalloids and colloids. Dung et al ${ }^{17}$ compared four intravenous fluid regimens (Ringer lactate, normal saline, $3 \%$ gelatin, and dextran 70) in 50 children aged 5-15 years with dengue shock; no difference was seen in the occurrence or duration of shock or fluid requirements in the three groups, and all patients recovered. However, the study was considered to be underpowered. In another study by Ngo et al, ${ }^{18}$ the same intravenous fluid regimens were studied in a larger cohort of 230 children with dengue, which included a larger proportion of patients with severe dengue. Again, no statistically significant benefit was seen with any regimen. However, a trend towards benefit of colloids over crystalloids was demonstrated, especially with early administration of colloids in more severely ill patients. In a third study, Wills et a ${ }^{19}$ compared three fluid regimens (Ringer lactate, dextran 70, and 6\% hydroxyethyl starch) in 512 children aged $2-15$ years with dengue shock. The authors stratified the study population into two groups, ie, moderate shock (pulse pressure $>10$ and $<20 \mathrm{mmHg}$ ) and severe shock (pulse pressure $<10 \mathrm{mmHg}$ ). Patients with moderate shock $(n=383)$ were randomized to receive Ringer lactate, dextran, or starch and those in severe shock $(n=129)$ were randomized to receive dextran or starch. No statistically significant differences were seen in either severity group in the requirement for colloid subsequent to the initial episode of shock, volumes of rescue colloid, total parenteral fluid administered, or number of days in the hospital. The authors concluded that treatment with colloids did not provide any benefit over treatment with Ringer lactate in patients with moderate shock. In patients with severe shock, no clear benefit with either starch or dextran was demonstrated. Despite the fact that there is no evidence to support the use of colloids in patients with severe shock, the authors felt that it would be unethical to compare colloids with crystalloids in such patients, because it is generally accepted that colloids are needed in cases of severe shock.

The ideal dose or rapidity with which fluid should be infused has not been demonstrated in the trials, and recommendations are based on treatment practices in centers which have had significant experience in treating dengue patients. ${ }^{9}$ Newer guidelines recommend more cautious use of fluids 
than was recommended in the past. With appropriate use of fluid resuscitation in the dengue shock state, mortality rates have been shown to be $<0.2 \%$. It is important to reduce intravenous fluids once the patient is recovering, because overhydration can result in intravascular fluid overload once the vascular permeability reverses with recovery.

The exact details and recommendations are available in guidelines published by the World Health Organization and local health authorities in endemic countries. ${ }^{9,14}$ It is not the aim of this review to re-explain them. However, the principles of fluid therapy can be summarized as follows:

- Fluid must be administered orally as much as possible. Intravenous supplementation is necessary when the patient is not able to take fluids orally (severe vomiting, prostration) or is in shock.

- The rationale in fluid management is to keep enough fluid in the vascular system during the leakage phase to avoid hypovolemia and while also avoiding overloading the patient with too much fluid.

- The recommended first-line intravenous fluid is crystalloids $(0.9 \%$ saline $) .{ }^{9}$

- The rate of intravenous fluid administration should be at stepwise increments or decrements with at least 4-6-hourly hematocrit monitoring during the critical phase. ${ }^{9}$

- However, in situations of shock, immediate resuscitation with $20 \mathrm{~mL} / \mathrm{kg}$ boluses is recommended until blood pressure is recordable.

- Some guidelines recommend the calculation of a fluid quota for the entire critical period. ${ }^{14}$ This involves calculation of the maintenance fluid requirement for 24 hours plus a $50 \mathrm{~mL} / \mathrm{kg}$ (up to $50 \mathrm{~kg}$ ) deficit correction and spreading this total amount over 48 hours. This entails the total amount of fluid (both intravenous and oral with boluses included) to be given over the 48 hours of the critical phase.

- Colloids (eg, dextran) are recommended as second-line therapy when hypotension is not responsive to boluses of intravenous crystalloids (with reference to local or World Health Organization guidance).

- A rise in hematocrit indicates further hemoconcentration due to leakage, and hence a need for more fluids. However a drop in hematocrit may be due to either convalescence (reabsorption of extravasated fluid) or internal bleeding. If the patient is still ill and in critical phase with low platelet counts, always suspect bleeding. Sometimes there may not be any overt external manifestations of bleeding. In cases of suspected bleeding, the management strategy is transfusion of fresh whole blood.
- Administration of fluids should be guided by frequent monitoring and assessment of intravascular volume status during the critical stage, and fluids should never be administered at a constant rate without monitoring.

\section{Blood products}

In practice, platelet transfusions are usually given to patients who develop serious hemorrhagic manifestations or have very low platelet counts, although the exact platelet count at which platelets should be given has not been defined. Transfused platelets survive only for a very short period in patients with shock syndrome. ${ }^{20}$ The degree of elevation of circulating platelets after transfusion varies directly with the amount of platelets transfused and inversely with the degree of shock. There is some evidence of benefit with fresh frozen plasma transfusion in increasing platelet counts, ${ }^{21}$ although the effect of plasma transfusion in dengue shock has not been studied in a controlled clinical trial. Blood transfusion is required in patients with severe hemorrhage, but there are no published data on its use.

\section{Recovery phase}

There is no need to restrict fluids as the patient recovers. The entire fluid amount can be given orally. However, close monitoring is necessary to recognize heart failure or pulmonary edema during recovery especially in patients with comorbidities such as congenital heart disease, ischemic heart disease, hypertension, and diabetes.

\section{Other aspects of management}

Dengue can have several other complications during the course of the illness, such as hepatitis, arthralgia, myocarditis, glomerulonephritis, and electrolyte imbalances, which are further complicated by fluid therapy. ${ }^{22,23}$ Therefore, liver function tests, renal function tests, electrolytes, electrocardiography, and echocardiography should be performed in dengue patients routinely and as needed (as resources and logistics permit). From a public health perspective, notification of cases and health education of the public will help to prevent further infections.

\section{Immunomodulation}

Given the postulated pathogenesis of dengue, treatments targeting the immune system have been trialed in dengue, with little success. The rationale for their use is to suppress the presumed immunological overactivity of the host in response to the virus. It is hypothesized that cytokines, including tumor necrosis factor alpha, interleukin (IL)-2, IL-6, IL-8, 
IL-10, IL-12, and interferon gamma are significantly elevated in severe dengue and cause damage to the endothelial cells of the capillaries, resulting in fluid leakage. ${ }^{24}$

\section{Corticosteroids}

The World Health Organization guidelines for management of dengue do not recommend the use of corticosteroids. Clinical trials of corticosteroids in dengue have been inconclusive so far, and for the most part have been underpowered and lacking in methodological quality. ${ }^{7}$ Some of the early studies demonstrated possible beneficial effects of corticosteroids in dengue shock. However, a Cochrane review of these studies showed no benefit. ${ }^{25}$ Nonetheless, these randomized studies have been small and were performed over 20 years ago. ${ }^{7}$ A more recent study showed no beneficial effect of dexamethasone on platelet counts. ${ }^{26,27}$ A recent retrospective study showed some benefit with methylprednisolone in patients with severe dengue. ${ }^{28}$ Nonetheless, although some clinicians use steroids in treatment, ${ }^{29,30}$ there is currently no clear evidence to justify the use of corticosteroids in the treatment of severe dengue. There is a clear need for adequately powered, randomized, double-blind, placebo-controlled clinical trials in both children and adults to evaluate fully the possible benefit or lack of benefit of corticosteroids in dengue infection.

\section{Intravenous immunoglobulins}

Similar to corticosteroids, intravenous immunoglobulins have been used in the treatment of dengue with little success. Ostronoff et $\mathrm{al}^{31}$ reported a series of five patients in Brazil with dengue and severe thrombocytopenia who were treated with intravenous immunoglobulins $(500 \mathrm{mg} / \mathrm{kg} /$ day infusion over 3 hours for 5 days). Clinical improvement, together with improvement in platelet count, was seen in these patients. However, the only published randomized controlled trial investigating the effect of intravenous immunoglobulins on thrombocytopenia showed no benefit. ${ }^{32}$ Seriously ill patients with hemorrhage or shock were excluded from that study and hence the possible effects of intravenous immunoglobulins on dengue shock state were not studied. One important conclusion was that intravenous immunoglobulins were safe, with no significant side effects being encountered during the trial. There is currently insufficient evidence to make any recommendation regarding the use of intravenous immunoglobulins in dengue shock, ${ }^{6}$ and further studies are required.

\section{Other supportive therapies}

A randomized controlled trial compared the use of nasal continuous positive airway pressure (NCPAP) versus oxygen by mask in 37 Vietnamese children with dengue shock state and acute respiratory failure. ${ }^{33}$ It was shown that NCPAP effectively decreased hypoxemia and reduced the number of children requiring intubation and ventilation. Thus, NCPAP appears to be an effective treatment in acute respiratory failure associated with dengue shock state.

Carbazochrome sodium sulfonate (AC-17), a hemostatic drug with a capillary-stabilizing action, has been shown to reduce the vascular hyperpermeability induced by vasoactive substances through an agonist-induced inhibition of phosphoinositide hydrolysis. ${ }^{34}$ Its effect in severe dengue was investigated in a small randomized clinical trial conducted in 95 Thai children. ${ }^{35}$ The primary outcome measure was prevention of capillary leakage, as evidenced by the presence of pleural effusion, and the secondary outcome was prevention of shock. No evidence of benefit in either outcome measure was seen using AC-17 for treatment of dengue shock state, although the study was underpowered to detect a potential treatment benefit.

The role of different inotropic and vasopressor agents in dengue shock has not been investigated in clinical trials. Vasopressor drugs such as noradrenaline and dopamine are indicated in shock that is unresponsive to fluids, but no clinical trials are available on their use in dengue. In the case of cardiac dysfunction, it is appropriate to use cardiac inotropic drugs such as dobutamine or adrenaline in combination with a vasopressor, although, again, no evidence is available.

\section{Issues in developing a vaccine for dengue}

As mentioned above, the dengue virus has four serologically distinct types (DV-1, DV-2, DV-3, and DV-4). The virus has a positive sense RNA strand coding for three structural proteins (C-capsid, E-envelope, and M-membrane) and seven nonstructural proteins (NS1, NS2a, NS2b, NS3, NS4a, NS4b, and NS5). ${ }^{36}$ Initial optimism about developing a successful vaccine for dengue centered on the simple structure of the virus and the successful development of a highly effective vaccine for yellow fever virus which shares structural similarities with the dengue virus.

However, several issues have prevented the development of a successful vaccine for dengue. The historical details of attempts to develop a dengue vaccine and its technical aspects are a vast subject and beyond the scope of this review. Instead, we concentrate on giving an overview of the obstacles encountered in making a dengue vaccine.

Firstly, unlike the yellow fever vaccine, the dengue virus has four distinct serotypes. They are all potentially lethal to 
humans in a severe infection. Therefore, the ideal vaccine must be immunogenic against all four serotypes rather than one.$^{37}$ It has been shown that infection with one serotype will offer long-term protection against that serotype, but probably only short-term protection against the others. ${ }^{38}$ In fact, for those getting a second infection with a different serotype, the severity may be greater (see below) ${ }^{39}$ Therefore, a theoretical risk exists in developing a monovalent vaccine because even if it is successful in confering immunity against one serotype, the risk may be greater for recipients if infected with a different serotype.$^{36}$ Therefore, current efforts are mostly directed towards developing a tetravalent vaccine with immunogenicity for all four serotypes. However, this is an economically and technically demanding task.

Secondly, our understanding of the pathophysiology of dengue fever is as yet incomplete. One theory as mentioned above is that a secondary heterotypic virus (a different dengue virus serotype) causes more severe infection by activating previously sensitized $\mathrm{T}$ and $\mathrm{B}$ cells and by interacting with circulating neutralizing antibodies for the previous serotype. It is hypothesized that this process activates an immune cascade and a cytokine storm that is ineffective in clearing the infecting virus serotype but is severely damaging to the host. ${ }^{40}$ However, certain observations preclude this being the only mechanism responsible (severe infection can occur as a first infection in individuals without any previously circulating antibodies, and infants without any pre-existing cellular immunity can have severe infection). ${ }^{41,42}$ The antibody-dependent enhancement theory initially proposed by Halstead (as the "original antigen sin") states that while the neutralizing antibodies of a previous infection are ineffective in combating current infection from a heterotypic virus, it still binds to these viruses and facilitates its uptake via $\mathrm{Fc}$ receptors to macrophages and monocytes, increasing target immune cell activation. This would obviously lead to an enhanced (and hazardous) immune response in the host. ${ }^{43,44}$ The implications of this phenomenon for development of a vaccine are that monovalent vaccines can be hazardous if they only produce neutralizing antibodies to a single serotype, and tetravalent vaccines must produce effective neutralizing antibody titers against all serotypes; suboptimal immune responses may again leave an individual vulnerable to a severe infection. Although these theoretical risks have not been realized in the limited clinical trials involving humans, in the event of a mass vaccination, this potentially is a cause for serious concern.

Thirdly, there is no animal model to recreate the disease process in humans. This is a major obstacle for vaccine development because preclinical trials cannot be clearly interpreted with regard to safety and efficacy. Many mammals, including mice, dogs, guinea pigs, and goats, do not develop an illness resembling dengue fever as seen when humans are inoculated with the virus. ${ }^{45}$ Nonhuman primates such as Rhesus and Cynomolgus monkeys (Macaca mulatta, Macaca fasicularis) develop viremia and an antibody response when inoculated via the subcutaneous route, but not the clinical disease. Nonetheless, many animal studies conducted to date on dengue vaccines are based on these primates. ${ }^{37,46,47}$

Fourthly, while neutralizing antibody titer following vaccination is a very important parameter for determining the efficacy and perhaps the safety of the vaccine, there is considerable difficulty in accurately assessing such titers with current assay methods. There is a lack of uniformity in assay methods employed in earlier vaccine trials, making it difficult to undertake a head-to-head comparison. ${ }^{36,48}$ Recently, the World Health Organization has made attempts to arrive at uniform protocols in assessment of dengue vaccine trials. ${ }^{45}$ There is also the difficulty in differentiating between different neutralizing antibodies to each serotype. This is further complicated by the fact that, in endemic regions, potential recipients may already harbor neutralizing antibodies to one or many serotypes, making it difficult to differentiate the response to the vaccine.

Fifthly, there are other concerns, some of which are theoretical (effect of a possible successful tetravalent vaccine on the wild-type dengue virus) and some are technical issues with regard to various vaccine platforms (nonuniformity of immune response in tetravalent live attenuated vaccines for each serotype, lack of immunogenicity, limited efficiency of uptake of plasmids of DNA vaccines by host immune cells, and finding a nonhazardous vector for viral vector-based DNA vaccines). ${ }^{36,45}$

All these issues have so far delayed or prevented the development of a successful vaccine against dengue and some of the obstacles are yet to be overcome. Despite these, research is still ongoing because the cost-effectiveness of an efficacious vaccine will be massive in all endemic areas. ${ }^{49}$ Many different vaccine platforms are currently being investigated and are in various stages of development. These include live attenuated virus, purified inactivated virus, a dengue virus-yellow fever chimeric, and subunitbased and DNA-based vaccines. ${ }^{45}$ Some have progressed to clinical trials in endemic areas involving small numbers of participants (adults, children, and infants). ${ }^{50-52}$ The fears of antibody-dependent enhancement in the recipients have not been realized and the long-term safety data are satisfactory 
in these trials. However, the protective immune response was not long-lasting or uniform across the different serotypes, making the trial vaccines fall short in efficacy. Therefore, the quest for a "perfect" dengue vaccine is still on, with different developers focusing on different vaccine platforms.

\section{Future directions}

One of the major areas for future research is development of the vaccines which has been discussed extensively in the preceding section. Research into the understanding of the pathophysiology of dengue, mainly the mechanisms behind the processes of capillary leakage and drop in platelet count, will help in accurate prediction of the critical phase, identifying at-risk patients, and targeting specific immunomodulatory therapy. The role of immunosuppressive/immunomodulatory therapy in dengue has not been adequately explored. As mentioned previously, the number of trials assessing the efficacy of steroids is small and has yielded conflicting results. ${ }^{7,25}$ Use of different preparations and doses of steroids makes it difficult to have a head-to-head comparison of study results. There is a need for a well-designed large-scale randomized clinical trial in this regard.

However, it must be acknowledged that there are certain difficulties in initiating and continuing with clinical trials involving immunomodulatory agents. Dengue is an acute and short-lasting infection. The harms and costs of such specific therapy have to be balanced against the expected benefit, even if the trials show that the agents are effective. For example, administration of intravenous immunoglobulins is clearly not economically feasible for many patients at the time of an epidemic, even if the trials showed that it is effective. ${ }^{6}$ Even in the absence of a specific therapy, in our clinical experience, supportive therapy in dengue is only effective when coupled with close monitoring. In such a situation it is difficult to justify subjecting patients with an acute short-lasting infection to potentially harmful and costly immunodulatory or immunosuppressive therapy in the setting of a trial. Selecting critically ill patients also creates an ethical dilemma because the presumed hypothetical benefits of such therapy cannot be based on solid reasoning, given that the pathophysiology of the disease itself is an enigma. Dengue in many endemic areas has become a sociopolitical issue because those who usually succumb to the illness are previously healthy and young individuals. Therefore, many clinicians and researchers are reluctant to go beyond the local guidelines (recommending effective supportive therapy) to try out new modes of specific therapy.
Despite the practical difficulties in conducting clinical trials on specific therapies and technical difficulties in creating a vaccine, we feel that an equally important and less controversial mode of controlling dengue should not be forgotten. That is the public health aspect of prevention of disease. Central health policy-making institutions of a country should have a detailed plan on case reporting, contact tracing, morbidity and mortality conferences, and environmental health improvement (grass-roots level teams to inspect local mosquito breeding sites in their localities, measures of vector control, and public health education). The legal framework should be strengthened to encourage citizens to keep their vicinities clear of mosquito breeding sites as much as possible. A successful dengue control campaign needs committed political backing to ensure adequate funding, central policy-making, supply and training of skilled personnel (entomologists, microscopists, health care workers) and an infrastructure (hospitals, drugs, laboratory facilities) to handle seasonal epidemics.

\section{Conclusion}

Dengue is a global health problem, with approximately 2.5 billion of the world's population at risk of infection. The incidence of dengue has risen over the last 50 years at a phenomenal rate for reasons which are as yet poorly explained. Despite being a short-lasting illness without any significant long-term sequelae, a severe infection can be lethal. The pathophysiology of dengue is still obscure, although it is widely accepted that the "severe illness" is due to an interaction between the virus and the over-reacting immune system of the host. There is no specific treatment for the infection, and management is only supportive care with judicious fluid management during the critical phase coupled with continuous monitoring. The advantages of having a vaccine against dengue are immense, but progression in this regard is slow due to many technical difficulties.

\section{Disclosure}

The authors report no conflicts of interest in this work.

\section{References}

1. Weaver SC, Vasilakis N. Molecular evolution of dengue viruses: contributions of phylogenetics to understanding the history and epidemiology of the preeminent arboviral disease. Infect Genet Evol. 2009;9: 523-540.

2. Thomas SJ, Strickman D, Vaughn DW. Dengue epidemiology: virus epidemiology, ecology, and emergence. Adv Virus Res. 2003;61: 235-289.

3. Sanchez V, Gimenez S, Tomlinson B, et al. Innate and adaptive cellular immunity in flavivirus-naive human recipients of a live-attenuated dengue serotype 3 vaccine produced in Vero cells (VDV3). Vaccine. 2006;24:4914-4926. 
4. Messer WB, Vitarana UT, Sivananthan K, et al. Epidemiology of dengue in Sri Lanka before and after the emergence of epidemic dengue hemorrhagic fever. Am J Trop Med Hyg. 2002;66:765-773.

5. Gupta E, Dar L, Kapoor G, Broor S. The changing epidemiology of dengue in Delhi, India. Virol J. 2006;3:92.

6. Rajapakse S. Intravenous immunoglobulins in the treatment of dengue illness. Trans R Soc Trop Med Hyg. 2009;103:867-870.

7. Rajapakse S. Corticosteroids in the treatment of dengue illness. Trans R Soc Trop Med Hyg. 2009;103:122-126.

8. Simasathien S, Watanaveeradej V. Dengue vaccine. J Med Assoc Thai. 2005;88 Suppl 3:S363-S377.

9. World Health Organization. Dengue: Guidelines for diagnosis, treatment, prevention and control. Available at: http://whqlibdoc.who.int/ publications/2009/9789241547871_eng.pdf. Accessed June 13, 2012.

10. Jelinek T. Trends in the epidemiology of dengue fever and their relevance for importation to Europe. Euro Surveill. 2009;14(25): 19250.

11. Centers for Disease Control and Prevention. Dengue fever and dengue hemorrhagic fever. Available at: http://wwwnc.cdc.gov/travel/ yellowbook/2012/chapter-3-infectious-diseases-related-to-travel/denguefever-and-dengue-hemorrhagic-fever.htm. Accessed June 13, 2012.

12. Kalayanarooj S, Vaughn DW, Nimmannitya S, et al. Early clinical and laboratory indicators of acute dengue illness. J Infect Dis. 1997;176:313-321.

13. Stephenson JR. Understanding dengue pathogenesis: implications for vaccine design. Bull World Health Organ. 2005;83:308-314.

14. Ministry of Health. National Guidelines on Management of Dengue Fever and Hemorrhagic Fever in adults and children. Colombo, Sri Lanka: Ministry of Health; 2011.

15. Premaratna R, Liyanaarachchi E, Weerasinghe M, de Silva HJ. Should colloid boluses be prioritized over crystalloid boluses for the management of dengue shock syndrome in the presence of ascites and pleural effusions? BMC Infect Dis. 2011;11:52.

16. Nimmannitya S. Clinical spectrum and management of dengue haemorrhagic fever. Southeast Asian J Trop Med Public Health. 1987;18:392-397.

17. Dung NM, Day NP, Tam DT, et al. Fluid replacement in dengue shock syndrome: a randomized, double-blind comparison of four intravenousfluid regimens. Clin Infect Dis. 1999;29:787-794.

18. Ngo NT, Cao XT, Kneen R, et al. Acute management of dengue shock syndrome: a randomized double-blind comparison of 4 intravenous fluid regimens in the first hour. Clin Infect Dis. 2001;32:204-213.

19. Wills BA, Nguyen MD, Ha TL, et al. Comparison of three fluid solutions for resuscitation in dengue shock syndrome. $N$ Engl J Med. 2005;353:877-889.

20. Isarangkura $\mathrm{P}$, Tuchinda $\mathrm{S}$. The behavior of transfused platelets in dengue hemorrhagic fever. Southeast Asian J Trop Med Public Health. 1993;24 Suppl 1:222-224.

21. Sellahewa KH, Samaraweera N, Thusita KP, Fernando JL. Is fresh frozen plasma effective for thrombocytopenia in adults with dengue fever? A prospective randomised double blind controlled study. Ceylon Med J. 2008;53:36-40.

22. Weerakoon KG, Kularatne SA, Edussuriya DH, et al. Histopathological diagnosis of myocarditis in a dengue outbreak in Sri Lanka, 2009. BMC Res Notes. 2011;4:268.

23. Ghosh M, Banerjee M, Das S, Chakraborty S. Dengue infection with multi-organ involvement. Scand J Infect Dis. 2011;43:316-318.

24. Kurane I. Dengue hemorrhagic fever with special emphasis on immunopathogenesis. Comp Immunol Microbiol Infect Dis. 2007;30: 329-340.

25. Panpanich R, Sornchai P, Kanjanaratanakorn K. Corticosteroids for treating dengue shock syndrome. Cochrane Database Syst Rev. 2006;3:CD003488.

26. Srichaikul T, Punyagupta S, Sorakhunpipitkul L, Udomsubpayakul U. Adjunctive corticosteroid therapy in 149 grade II (non-shock) adult DHF patients: an analysis during January 2008-February 2010. J Med Assoc Thai. 2011;94:1419-23.
27. Kularatne SA, Walathara C, Mahindawansa SI, et al. Efficacy of low dose dexamethasone in severe thrombocytopenia caused by dengue fever: a placebo controlled study. Postgrad Med J. 2009;85:525-529.

28. Premaratna R, Jayasinghe KG, Liyanaarachchi EW, Weerasinghe OM, Pathmeswaran A, de Silva HJ. Effect of a single dose of methyl prednisolone as rescue medication for patients who develop hypotensive dengue shock syndrome during the febrile phase: a retrospective observational study. Int J Infect Dis. 2011;15:e433-e434.

29. Kularatne SA. Survey on the management of dengue infection in Sri Lanka: opinions of physicians and pediatricians. Southeast Asian JTrop Med Public Health. 2005;36:1198-1200.

30. Rajapakse S, Ranasinghe C, Rodrigo C. Corticosteroid therapy in dengue infection - opinions of junior doctors. J Glob Infect Dis. 2010;2:199-200.

31. Ostronoff M, Ostronoff F, Florencio R, et al. Serious thrombocytopenia due to dengue hemorrhagic fever treated with high dosages of immunoglobulin. Clin Infect Dis. 2003;36:1623-1624.

32. Dimaano EM, Saito M, Honda S, et al. Lack of efficacy of high-dose intravenous immunoglobulin treatment of severe thrombocytopenia in patients with secondary dengue virus infection. Am J Trop Med Hyg. 2007;77:1135-1138.

33. Cam BV, Tuan DT, Fonsmark L, et al. Randomized comparison of oxygen mask treatment vs nasal continuous positive airway pressure in dengue shock syndrome with acute respiratory failure. J Trop Pediatr. 2002;48:335-339.

34. Sendo T, Itoh Y, Aki K, Oka M, Oishi R. Carbazochrome sodium sulfonate (AC-17) reverses endothelial barrier dysfunction through inhibition of phosphatidylinositol hydrolysis in cultured porcine endothelial cells. Naunyn Schmiedebergs Arch Pharmacol. 2003;368:175-180.

35. Tassniyom S, Vasanawathana S, Dhiensiri T, Nisalak A, Chirawatkul A. Failure of carbazochrome sodium sulfonate (AC-17) to prevent dengue vascular permeability or shock: a randomized, controlled trial. J Pediatr. 1997; 131:525-528.

36. Thomas SJ, Endy TP. Critical issues in dengue vaccine development. Curr Opin Infect Dis. 2011;24:442-450.

37. Raviprakash K, Wang D, Ewing D, et al. A tetravalent dengue vaccine based on a complex adenovirus vector provides significant protection in rhesus monkeys against all four serotypes of dengue virus. $J$ Virol. 2008;82:6927-6934.

38. Sabin AB. Research on dengue during World War II. Am J Trop Med Hyg. 1952;1:30-50.

39. Halstead SB. Epidemiological studies of Thai haemorrhagic fever. Bull World Health Organ. 1966;35:80-81.

40. Rothman AL. Cellular immunology of sequential dengue virus infection and its role in disease pathogenesis. Curr Top Microbiol Immunol. 2010;338:83-98.

41. Lin CC, Huang YH, Shu PY. Characteristic of dengue disease in Taiwan. Am J Trop Med Hyg. 2010;82:731-739.

42. Kliks SC, Nimmannitya S, Nisalak A, Burke DS. Evidence that maternal dengue antibodies are important in the development of dengue hemorrhagic fever in infants. Am J Trop Med Hyg. 1988;38:411-419.

43. Halstead SB, Mahalingam S, Marovich MA. Intrinsic antibody-dependent enhancement of microbial infection in macrophages: disease regulation by immune complexes. Lancet Infect Dis. 2010;10:712-722.

44. Halstead SB, Nimmannitya S, Cohen SN. Observations related to pathogenesis of dengue hemorrhagic fever. IV. Relation of disease severity to antibody response and virus recovered. Yale J Biol Med. 1970;42:311-328.

45. Raviprakash K, Defang G, Burgess T, Porter K. Advances in dengue vaccine development. Hum Vaccin. 2009;5:520-528.

46. Chen L, Ewing D, Subramanian H, et al. A heterologous DNA primeVenezuelan equine encephalitis virus replicon particle boost dengue vaccine regimen affords complete protection from virus challenge in cynomolgus macaques. J Virol. 2007;81:11634-11639.

47. Raviprakash K, Apt D, Brinkman A, et al. A chimeric tetravalent dengue DNA vaccine elicits neutralizing antibody to all four virus serotypes in rhesus macaques. Virology. 2006;353:166-173. 
48. Thomas SJ. The necessity and quandaries of dengue vaccine development. J Infect Dis. 2011;203:299-303.

49. Lee BY, Connor DL, Kitchen SB, et al. Economic value of dengue vaccine in Thailand. Am J Trop Med Hyg. 2011;84:764-772.

50. Simasathien S, Thomas SJ, Watanaveeradej V, et al. Safety and immunogenicity of a tetravalent live-attenuated dengue vaccine in flavivirus naive children. Am J Trop Med Hyg. 2008;78:426-433.
51. Watanaveeradej V, Simasathien S, Nisalak A, et al. Safety and immunogenicity of a tetravalent live-attenuated dengue vaccine in flavivirusnaive infants. Am J Trop Med Hyg. 2011;85:341-351.

52. Sabchareon A, Lang J, Chanthavanich P, Yoksan S, Forrat R, Attanath P. Safety and immunogenicity of tetravalent live-attenuated dengue vaccines in Thai adult volunteers: role of serotype concentration, ratio and multiple doses. Am J Trop Med Hyg. 2002;66:264-272.

\section{Publish your work in this journal}

Infection and Drug Resistance is an international, peer-reviewed openaccess journal that focuses on the optimal treatment of infection (bacterial, fungal and viral) and the development and institution of preventive strategies to minimize the development and spread of resistance. The journal is specifically concerned with the epidemiology of antibiotic

\section{Dovepress}

resistance and the mechanisms of resistance development and diffusion in both hospitals and the community. The manuscript management system is completely online and includes a very quick and fair peerreview system, which is all easy to use. Visit http://www.dovepress.com/ testimonials.php to read real quotes from published authors.

Submit your manuscript here: http://www.dovepress.com/infection-and-drug-resistance-journal 